IISc-CTS-1/98

hep-ph/9802338

\title{
The Low Energy Expansion for Pion-Pion Scattering and Crossing Symmetry in Dispersion Relations
}

\author{
B. Ananthanarayan \\ Centre for Theoretical Studies, \\ Indian Institute of Science, \\ Bangalore 560 012, India.
}

\begin{abstract}
We show that a suitable setting for comparison of the low-energy representation for pion-pion scattering amplitudes, with dispersive representation for these amplitudes, is provided by certain manifestly crossing symmetric dispersion relations. We begin with a discussion of fixed-t dispersion relations and discuss the origin of crossing constraints that arise in this context when we consider resonance saturation with certain $l \geq 2$ states. We demonstrate that the approach advocated here does not require us to enforce such constraints. Our results are contrasted with those from fixed-t dispersion relations. We finally discuss the numerical import of our results.
\end{abstract}

PACS Number(s): 11.55.Fv, 11.80.Et, 13.75.Lb, 12.39.Fe 


\section{Introduction}

Chiral perturbation theory[酐 provides a low-energy representation for pion-pion scattering amplitudes that are manifestly crossing symmetric and have well-known analyticity properties. In particular, to $O\left(p^{6}\right)$ in the momentum expansion (with the pion mass $m_{\pi}^{2}$ treated as a quantity of $\left.O\left(p^{2}\right)\right)$ the pion-pion scattering amplitude may be written down in terms of three functions of single variables $W_{I}(s), I=0,1,2[2]$. One may then compute the low-energy expansion in the energies and pion masses in terms of these functions which have now been explicitly computed 3 , 4. Furthermore, the detailed knowledge of the amplitude in field theory with standard power counting [4, 5], is expected to lead to accurate predictions for the $\mathrm{I}=0 \mathrm{~S}$ - wave scattering length $a_{0}^{0}$. This important physical quantity, whose reported value is $0.26 \pm 0.05[6]$ has been measured indirectly only at the level of $20 \%$ accuracy and is expected to be measured to greater precision [7].

Pion-pion scattering is a problem that is particular well suited to dispersion relation analysis 8], rigorously established in axiomatic field theory. One approach has been to write down fixed-t dispersion relations, with two subtractions which suffice to guarantee convergence as a consequence of the Froissart bound, for amplitudes of definite iso-spin. It has been recognized for a long time that crossing symmetry in dispersion relations brings in many subtle considerations. It is possible to enforce crossing symmetry partially to eliminate the unknown t-dependent functions in favor of the two S- wave scattering lengths[9]. We note that it is the requirement of two subtractions in the dispersion relations that makes the accurate determination of the S- wave scattering amplitudes from dispersion relations problematic — one needs to combine theoretical inputs with phenomenology in 
order to extract precise predictions for these quantities [4, 5, 10].

The two subtractions in the dispersion relations we consider, leave the $\mathrm{S}$ - and $\mathrm{P}$ - wave absorptive parts completely unconstrained [1] by crossing symmetry. One may write down the total amplitude as (a) a part that is saturated only in terms of these absorptive parts, and (b) a part that is saturated by the $l \geq 2$ absorptive parts. In Ref. [12] we explictly showed that (a) may be expressed in a manner whereby the dispersive representation of the low-energy polynomials in terms of these absorptive parts may be explicitly produced. Here we discuss case (b) at some length.

Our analysis begins with considering fixed-t dispersion relations. We must expand the Roy version of fixed-t dispersion relations saturated with certain $l \geq 2$ absorptive parts, which yield the dispersive representation for the low energy polynomials in the energies and pion masses. We immediately encounter problems that arise from the inadequate implementing of crossing symmetry by fixed-t disperion relations, which we discuss at some length. Results analogous to (a) as well as (b) with fixed-t dispersion relations have also been discussed elsewhere [3, 13]. The numerical import of these shows clearly that any attempt to saturate the dispersion relations with the absorptive parts of two of the well-known resonances listed by the Particle Data Group[14], the $I=0, l=2$ state $f_{2}(1270)$ (hereafter referred to as the $f$ ) and the $I=1, l=3$ state $\rho_{3}(1690)$ (hereafter refered to as the $g$ ) violates crossing symmetry - restoration of crossing symmetry with these would predict a $\pi \pi$ width of a nearly $110 \mathrm{MeV}$ for the $g$, which falls short by a factor of almost 3 .

We therefore advocate a different approach to (b), which overcomes the problems posed by crossing contraints. This is achieved by considering certain manifestly crossing 
symmetric dispersion relations. We compute with the $l \geq 2$ absorptive parts given by the $f$ and $g$, what is the manifestly crossing symmetric dispersive representation for the lowenergy polynomials in this approximation, which constitutes the central result of this work. The technique may be easily extended to other resonances and also to other models of partial waves.

The manifestly crossing symmetric dispersion relations are of the type first considered by Wanders 15 in the context of $\pi^{0} \pi^{0}$ scattering and subsequently by the authors of Ref. [16] for other totally symmetric amplitudes. The family of dispersion relations we consider is parameterized in terms of a variable $x_{0}$; we concentrate mainly on the special case of $x_{0}=0$. Note that crossing constraints on the $l \geq 2$ absorptive parts cannot disappear altogether: whereas for a fixed value of $x_{0}$ the representation does not entail any crossing constraints, as $x_{0}$ is varied, relations are implied on the absorptive parts of the $l \geq 2$ absorptive parts. However, one of our significant findings is that the low-energy polynomials of the type we are interest in, can be produced only with $x_{0}=0$.

We finally note that in a recent work [17], crossing symmetric dispersion relations have been employed to fix some of the chiral coupling constants by comparing the Taylor coefficients of the dispersive and chiral representations of the pion-pion scattering amplitudes at the symmetry point $s=t=u=4 / 3$ in the Mandelstam plane.

In Section 2 we will review the basics of pion-pion scattering and remark on the structure of the low-energy expansion. In Section 3 we will consider the form of fixed-t dispersion relations suitable for saturation with resonances and discuss the deviations from crossing symmetry. In Section 4 we will describe the method of writing down manifestly crossing symmetric dispersion relations and evaluate the manifestly crossing symmetric 
low-energy polynomials. In Section 5 we discuss the numerical import of our work. In Section 6 our conclusions are presented.

\section{Pion-Pion Scattering and The Low Energy Expansion}

Pion-pion scattering :

$$
\pi^{a}\left(p_{1}\right)+\pi^{b}\left(p_{2}\right) \rightarrow \pi^{c}\left(p_{3}\right)+\pi^{d}\left(p_{4}\right)
$$

where $a, b, c, d$ label the iso-spin and the $p_{i}, i=1,2,3,4$, are all incoming momenta, may be described in terms of a unique function $A(s, t, u)$, where $s, t, u$ stand for dimensionless Mandelstam variables,

$$
s=\left(p_{1}+p_{2}\right)^{2} / m_{\pi}^{2}, t=\left(p_{1}+p_{3}\right)^{2} / m_{\pi}^{2}, \text { and } u=\left(p_{1}+p_{4}\right)^{2} / m_{\pi}^{2},
$$

with $s+t+u=4$. We shall introduce units of energy squared where ever necessary in terms of the square of the pion mass, $m_{\pi}^{2}$. The choice of dimensionless squares of energies is very convenient in producing the low-energy expansion: we expand out the integrands of interest in the dispersive representations out in powers of $m_{\pi}^{2}$.

Since the strong interactions conserve iso-spin, it is customary to write down amplitudes of definite iso-spin in the s-channel:

$$
\begin{gathered}
T^{0}(s, t, u)=3 A(s, t, u)+A(t, u, s)+A(u, s, t) \\
T^{1}(s, t, u)=A(t, u, s)-A(u, s, t) \\
T^{2}(s, t, u)=A(t, u, s)+A(u, s, t)
\end{gathered}
$$

The scattering lengths $a_{l}^{I}$ arise in the threshold expansion for the partial wave amplitudes $\operatorname{Re} f_{l}^{I}(\nu)=\nu^{l}\left(a_{l}^{I}+b_{l}^{I} \nu+\ldots\right)$, where the partial wave expansion is given by $T^{I}(s, t, u)=$ 
$32 \pi \sum(2 l+1) f_{l}^{I}(s) P_{l}((t-u) /(s-4)), \nu=(s-4) / 4$ [the normalization of the amplitudes here differs with that of [16] by $32 \pi]$. The $b_{l}^{I}$ are the effective ranges.

We present our results for the case of saturating the dispersive integrals with the absorptive parts due to the $\rho(770)$ (hereafter referred to as the $\rho$ ), $f$ and $g$ in the narrow width approximation. (We consider the well-known case of the $\rho$ once more, for two reasons. Firstly, since the presence of two subtractions in the dispersion relations of interest does not constrain the absorptive part of the $\rho$ from crossing symmetry and our results will be identical whether we consider fixed-t dispersion relations or crossing symmetric ones. Secondly, the scale of the contribution to the low-energy coupling constants, or alternatively the $\beta_{i}$, from the S- and $\mathrm{P}$ - wave absorptive parts extracted from phenomenology and Roy equation fits to phase shift and elasticity information, is set by the $\rho$ as it is the dominant phenomenon in the $\mathrm{P}$ - wave channel in the low to medium energy.) The quantum numbers for these states as well as their physical properties are listed in Table 1[14]. The s-channel absorptive parts due to these resonances in the narrow width approximation:

$$
A^{I}(x, t)=32 \pi \sum_{l}(2 l+1) \operatorname{Im} f_{l}^{I}(x) P_{l}\left(1+\frac{2 t m_{\pi}^{2}}{x-4 m_{\pi}^{2}}\right)
$$

and the narrow width approximation reading

$$
\operatorname{Im} f_{l}^{I}(x)=\sqrt{\frac{x}{x-4 m_{\pi}^{2}}} \pi \Gamma_{m}^{\pi \pi} M_{m} \delta\left(x-M_{m}^{2}\right)
$$

Note that in the narrow-width approximation, the partial waves, despite being functions of only the variable $x$ do exhibit the correct normal threshold behaviour. We will have to keep this in mind when expanding out the integrands in our dispersive representations. It must also be noted that the arguments of the Legendre polynomials do contain $m_{\pi}^{2}$ since 
they could contain information of the external energies, parametrized by the dimensionless Mandelstam variables.

The low-energy representation for the pion-pion scattering amplitude $A(s, t, u)$, computed to two-loop accuracy in chiral perturbation theory, may be written down in terms of three functions of single variables, $W_{I}, I=1,2,3$ :

$$
A(s, t, u)=32 \pi\left\{\frac{1}{3} W_{0}(s)+\frac{3}{2}(s-u) W_{1}(t)+\frac{3}{2}(s-t) W_{1}(u)+\frac{1}{2} W_{2}(t)+\frac{1}{2} W_{2}(u)-\frac{1}{3} W_{2}(s)\right\}
$$

These functions are known to have only right hand cuts for $s>4$ and one then has the dispersion relations for these functions:

$$
\begin{gathered}
W_{0}(s)=A_{0}+B_{0} s+C_{0} s^{2}+D_{0} s^{3}+\frac{s^{4}}{\pi} \int_{4}^{\infty} \frac{d y}{y^{4}(y-s)} \operatorname{Im} W_{0}(y) \\
W_{1}(s)=A_{1}+B_{1} s+C_{1} s^{2}+\frac{s^{3}}{\pi} \int_{4}^{\infty} \frac{d y}{y^{3}(y-s)} \operatorname{Im} W_{2}(y) \\
W_{2}(s)=A_{2}+B_{2} s+C_{2} s^{2}+D_{2} s^{3}+\frac{s^{4}}{\pi} \int_{4}^{\infty} \frac{d y}{y^{4}(y-s)} \operatorname{Im} W_{2}(y)
\end{gathered}
$$

The coefficients $A_{I}, B_{I}, C_{I}, D_{I}, I=0,2$, and $A_{1}, B_{1}, C_{1}$ are the expansion coefficients of the functions $W_{I}$ and $W_{1}$ evaluated at $s=0$, and the amplitude $A(s, t, u)$ may be written most generally as:

$$
\beta_{1}+\beta_{2} s+\beta_{3} s^{2}+\beta_{4}(t-u)^{2}+\beta_{5} s^{3}+\beta_{6} s(t-u)^{2}+\text { integral. }
$$

The $\beta_{i}$ are known combinations of the these Taylor coefficients. It has been shown that the integral in the expression above has the same structure as that obtained from dispersion relations with two subtractions with the neglect of all but the absorptive parts of the Sand $\mathrm{P}$ - waves. In particular, the $\beta_{i}$ are completely expressible in terms of the two S- wave scattering lengths and certain integrals over the S- and $\mathrm{P}$ - wave absorptive parts [12], in 
this approximation. Nevertheless, the absorptive parts of the $l \geq 2$ states do contribute to the $\beta_{i}$ 's at this order, although they do not contribute to the integral in eq. 2.10) [2, 33, 13. The objective of the present work is, of course, to compute these contributions for certain $l \geq 2$ absorptive parts.

\section{Fixed-t Dispersion Relations}

A very convenient form of dispersion relations has been found [9] which eliminates the unknown functions $\mu_{I}, \nu_{I}$ in favour of the S- wave scattering lengths $a_{0}^{0}$ and $a_{0}^{2}$, where This (Roy) form is:

$$
\begin{aligned}
T^{I}(s, t) & =\sum_{I^{\prime}} \frac{1}{4}\left(s \mathbf{1}^{I I^{\prime}}+t C_{s t}^{I I^{\prime}}+u C_{s u}^{I I^{\prime}}\right) T_{s}^{I^{\prime}}(4,0) \\
& +\int_{4 m_{\pi}^{2}}^{\infty} d x g_{2}^{I I^{\prime}}(s, t, x) A^{I^{\prime}}(x, 0)+\int_{4 m_{\pi}^{2}}^{\infty} d x g_{3}^{I I^{\prime}}(s, t, x) A^{I^{\prime}}(x, t) .
\end{aligned}
$$

where the crossing matrices $C_{s t}, C_{s u}$ and $C_{t u}$ are defined below:

$$
\begin{gathered}
C_{s t}=\left[\begin{array}{ccc}
\frac{1}{3} & 1 & \frac{5}{3} \\
\frac{1}{3} & \frac{1}{2} & -\frac{5}{6} \\
\frac{1}{3} & -\frac{1}{2} & \frac{1}{6}
\end{array}\right], C_{s u}=\left[\begin{array}{ccc}
\frac{1}{3} & -1 & \frac{5}{3} \\
-\frac{1}{3} & \frac{1}{2} & \frac{5}{6} \\
\frac{1}{3} & \frac{1}{2} & \frac{1}{6}
\end{array}\right], \\
C_{t u}=\left[\begin{array}{ccc}
1 & 0 & 0 \\
0 & -1 & 0 \\
0 & 0 & 1
\end{array}\right],
\end{gathered}
$$

and $T^{I}(4,0)=32 \pi a_{0}^{I}$, and will play no role in the present discussion. For our purposes, it is convenient to write the kernels in the form

$$
\begin{aligned}
g_{2}(s, t, x) & =-\frac{t m_{\pi}^{2}}{\pi x\left(x-4 m_{\pi}^{2}\right)}\left(u m_{\pi}^{2} C_{s t}+s m_{\pi}^{2} C_{s t} C_{t u}\right)\left(\frac{\mathbf{1}}{x-t m_{\pi}^{2}}+\frac{C_{s u}}{x-s m_{\pi}^{2}-u m_{\pi}^{2}}\right) \\
g_{3}(s, t, x) & =-\frac{s u m_{\pi}^{4}}{\pi x\left(x-4 m_{\pi}^{2}+t m_{\pi}^{2}\right)}\left(\frac{\mathbf{1}}{x-s m_{\pi}^{2}}+\frac{C_{s u}}{x-u m_{\pi}^{2}}\right) .
\end{aligned}
$$


The contribution from the $\rho$ to $A(s, t, u)$ is unambiguous 18 and corresponds to precisely the form of the amplitude presented in Appendix $\mathrm{C}$ of Ref.[1], as well as with the dispersion relation evaluation presented in Ref.[19]:

$$
48 \pi \Gamma_{\rho}^{\pi \pi} \sqrt{\frac{M_{\rho}^{2}}{M_{\rho}^{2}-4 m_{\pi}^{2}}} \frac{1}{M_{\rho}\left(M_{\rho}^{2}-4 m_{\pi}^{2}\right)} m_{\pi}^{4}\left(\frac{t(s-u)}{M_{\rho}^{2}-t m_{\pi}^{2}}+\frac{u(s-t)}{M_{\rho}^{2}-u m_{\pi}^{2}}\right)
$$

Retaining the kinematic factor that ensures normal threshold behaviour, we write the amplitude out to the required order below as:

$$
\begin{gathered}
A(s, t, u)_{\rho}=\frac{12 \pi \Gamma_{\rho}^{\pi \pi}}{M_{\rho}^{7}} \sqrt{\frac{M_{\rho}^{2}}{M_{\rho}^{2}-4 m_{\pi}^{2}}} m_{\pi}^{4}\left(-32 M_{\rho}^{2}-192 m_{\pi}^{2}+32 M_{\rho}^{2} s+208 m_{\pi}^{2} s-\right. \\
\left.6 M_{\rho}^{2} s^{2}-52 m_{\pi}^{2} s^{2}+2 M_{\rho}^{2}(t-u)^{2}+12 m_{\pi}^{2}(t-u)^{2}+3 m_{\pi}^{2} s^{3}+m_{\pi}^{2} s(t-u)^{2}\right)
\end{gathered}
$$

In order to generate the dispersive representation for the low-energy polynomial, we merely expand the functions $g_{2}, g_{3}$ and the Legendre Polynomials out in powers of $m_{\pi}^{2}$. A general analysis of the framework considered here has been discussed earlier [3, 13] and will be discussed elsewhere as well[10].

The contribution of the $f$ to the iso-spin amplitudes resulting from the fixed-t (FT) dispersion relations, to this order read:

$$
\begin{gathered}
T^{0}(s, t, u)_{f}^{F T}=\frac{40 \pi \Gamma_{f}^{\pi \pi}}{3 M_{f}^{7}} \sqrt{\frac{M_{f}^{2}}{M_{f}^{2}-4 m_{\pi}^{2}}} m_{\pi}^{4}\left(-32 M_{f}^{2}-192 m_{\pi}^{2}-48 M_{f}^{2} s-560 m_{\pi}^{2} s\right. \\
\left.+14 M_{f}^{2} s^{2}+204 m_{\pi}^{2} s^{2}+2 M_{f}^{2}(t-u)^{2}+12 m_{\pi}^{2}(t-u)^{2}-13 m_{\pi}^{2} s^{3}+21 m_{\pi}^{2} s(t-u)^{2}\right) \\
T^{1}(s, t, u)_{f}^{F T}=\frac{40 \pi \Gamma_{f}^{\pi \pi}}{3 M_{f}^{7}} \sqrt{\frac{M_{f}^{2}}{M_{f}^{2}-4 m_{\pi}^{2}}} m_{\pi}^{4}\left(96 m_{\pi}^{2} s-16 m_{\pi}^{2}(t-u)-4 M_{f}^{2}(t-u) s\right. \\
\left.-24 m_{\pi}^{2}(t-u) s-48 m_{\pi}^{2} s^{2}+3 m_{\pi}^{2}(t-u) s^{2}-6 m_{\pi}^{2}(t-u)^{2} s+6 m_{\pi}^{2} s^{3}+m_{\pi}^{2}(t-u)^{3}\right) \\
T^{2}(s, t, u)_{f}^{F T}=\frac{40 \pi \Gamma_{f}^{\pi \pi}}{3 M_{f}^{7}} \sqrt{\frac{M_{f}^{2}}{M_{f}^{2}-4 m_{\pi}^{2}}} m_{\pi}^{4}\left(-32 M_{f}^{2}-192 m_{\pi}^{2}+2 M_{f}^{2}(t-u)^{2}-80 m_{\pi}^{2} s\right. \\
\left.-12 m_{\pi}^{2}(t-u)^{2}+3 m_{\pi}^{2}(t-u)^{2} s+2 M_{f}^{2} s^{2}+60 m_{\pi}^{2} s^{2}-7 m_{\pi}^{2} s^{3}\right)
\end{gathered}
$$


The contribution of the $g$ to the iso-spin amplitudes resulting from the fixed-t dispersion relations, to this order read:

$$
\begin{gathered}
T^{0}(s, t, u)_{g}^{F T}=\frac{56 \pi \Gamma_{g}^{\pi \pi}}{M_{f}^{7}} \sqrt{\frac{M_{g}^{2}}{M_{g}^{2}-4 m_{\pi}^{2}}} m_{\pi}^{4}\left(-32 M_{g}^{2}-192 m_{\pi}^{2}+32 M_{g}^{2} s+368 m_{\pi}^{2} s\right. \\
\left.-6 M_{g}^{2} s^{2}-132 m_{\pi}^{2} s^{2}+2 M_{g}^{2}(t-u)^{2}+12 m_{\pi}^{2}(t-u)^{2}+13 m_{\pi}^{2} s^{3}-9 m_{\pi}^{2} s(t-u)^{2}\right) \\
T^{1}(s, t, u)_{g}^{F T}=\frac{28 \pi \Gamma_{g}^{\pi \pi}}{M_{f}^{7}} \sqrt{\frac{M_{g}^{2}}{M_{g}^{2}-4 m_{\pi}^{2}}} m_{\pi}^{4}\left(-480 m_{\pi}^{2} s-16 m_{\pi}^{2}(t-u)+12 M_{g}^{2}(t-u) s+72 .\right. \\
\left.m_{\pi}^{2}(t-u) s+240 m_{\pi}^{2} s^{2}+30 m_{\pi}^{2}(t-u)^{2} s+3 m_{\pi}^{2}(t-u) s^{2}-30 m_{\pi}^{2} s^{3}+m_{\pi}^{2}(t-u)^{3}\right) \\
T^{2}(s, t, u)_{g}^{F T}=-\frac{1}{2} T^{0}(s, t, u)_{g}^{F T}
\end{gathered}
$$

Note that the factors $\sqrt{M_{m}^{2} /\left(M_{m}^{2}-4 m_{\pi}^{2}\right)}, m=f, g$ appear in the expressions for the amplitudes retaining the memory of the normal threshold behaviour of the partial waves.

It may be seen from the Roy form of the fixed-t dispersion relations eq.(3.1), that the amplitudes obtained at $O\left(p^{4}\right)$ are manifestly crossing symmetric and thus there are no crossing constraints at this order. However, at $O\left(p^{6}\right)$, we immediately observe the violation of crossing symmetry by considering, say $T^{1}(s, t, u)_{m}^{F T}$, which for $m=f, g$ contains terms that are even in powers of $(t-u)$. Note for instance the term in $T^{1}(s, t, u)_{f}^{F T}$,

$$
-240 \pi \Gamma_{f}^{\pi \pi} /\left(3 M_{f}^{7}\right) \sqrt{M_{f}^{2} /\left(M_{f}^{2}-4 m_{\pi}^{2}\right)} m_{\pi}^{6}(t-u)^{2} s
$$

and the term in $T^{1}(s, t, u)_{g}^{F T}$,

$$
840 \pi \Gamma_{g}^{\pi \pi} /\left(M_{g}^{7}\right) \sqrt{M_{g}^{2} /\left(M_{g}^{2}-4 m_{\pi}^{2}\right)} \cdot m_{\pi}^{6}(t-u)^{2} s
$$

One might then hope that if indeed the width of the $g$ were to be

$$
\Gamma_{g}^{\pi \pi}=\frac{2}{21}\left(\frac{M_{g}}{M_{f}}\right) \sqrt[6]{\frac{M_{g}^{2}-4 m_{\pi}^{2}}{M_{f}^{2}-4 m_{\pi}^{2}}} \Gamma_{f}^{\pi \pi}
$$


then these and possibly other inconvenient terms would be cancelled. It turns out that at $O\left(p^{6}\right)$ there is, in fact, only one crossing constraint [3, 13] which is the one given by eq.(3.12) as we show below.

We may wish to enforce the direct constraint on absorptive parts written down by Roy, which is a three component equation, for $I=0,1,2$ :

$$
\begin{gathered}
\int_{4 m_{\pi}^{2}}^{\infty} d x\left[\sum_{I^{\prime}}\left\{g_{2}^{I I^{\prime}}(s, t, x)-\left(C_{t u} g_{2}(s, u, x)\right)^{I I^{\prime}}\right\} A^{I^{\prime}}(x, 0)+\right. \\
\left.\left\{g_{3}^{I I^{\prime}}(s, t, x) A^{I^{\prime}}(x, t)-\left(C_{t u} g_{3}(s, u, x)\right)^{I I^{\prime}} A^{I^{\prime}}(x, u)\right\}\right]=0
\end{gathered}
$$

The left hand side of this equation when saturated with the absorptive part due to the $f$ in the narrow width approximation and worked out to $O\left(p^{6}\right)$, yields the result

$$
\frac{640 \pi \Gamma_{f}}{M_{f}^{7}} \sqrt{\frac{M_{f}^{2}}{M_{f}^{2}-4 m_{\pi}^{2}}} m_{\pi}^{6} s t u \delta^{I 1}
$$

while the result for the $g$ yields

$$
-\frac{6720 \pi \Gamma_{g}}{M_{g}^{7}} \sqrt{\frac{M_{g}^{2}}{M_{g}^{2}-4 m_{\pi}^{2}}} m_{\pi}^{6} s t u \delta^{I 1} .
$$

This reproduces the result eq.(3.12) if these two states were to guarantee crossing symmetry at this order.

We define below the combination $\left(T^{0}(s, t, u)_{m}^{F T}-T^{2}(s, t, u)_{m}^{F T}\right) / 3$ and define it to be equal to $A(s, t, u)_{m}^{F T}$ (despite the fact that crossing symmetry is not respected) to obtain the expressions below:

$$
A(s, t, u)_{f}^{F T}=\frac{80 \pi \Gamma_{f}^{\pi \pi}}{3 M_{f}^{7}} \sqrt{\frac{M_{f}^{2}}{M_{f}^{2}-4 m_{\pi}^{2}}} m_{\pi}^{4} s\left(-8 M_{f}^{2}-80 m_{\pi}^{2}+2 M_{f}^{2} s+24 m_{\pi}^{2} s-m_{\pi}^{2} s^{2}+3 m_{\pi}^{2}(t-u)^{2}\right),
$$


and

$$
\begin{gathered}
A(s, t, u)_{g}^{F T}=\frac{28 \pi \Gamma_{g}^{\pi \pi}}{M_{g}^{7}} \sqrt{\frac{M_{g}^{2}}{M_{g}^{2}-4 m_{\pi}^{2}}} m_{\pi}^{4}\left(-32 M_{g}^{2}-192 m_{\pi}^{2}+32 M_{g}^{2} s+368 m_{\pi}^{2} s-\right. \\
\left.6 M_{g}^{2} s^{2}-132 m_{\pi}^{2} s^{2}+2 M_{g}^{2}(t-u)^{2}+12 m_{\pi}^{2}(t-u)^{2}+13 m_{\pi}^{2} s^{3}-9 m_{\pi}^{2} s(t-u)^{2}\right)(3.1
\end{gathered}
$$

\section{Crossing Symmetric Dispersion Relations}

In Ref. [16], a remarkable dispersive representation is provided for a system of three totally symmetric amplitudes, $G_{0}, G_{1}$ and $G_{2}$ constructed out of the three iso-spin amplitudes that we are familiar with. In particular, $G_{0}$ is the $\pi^{0} \pi^{0}$ scattering amplitude. $G_{1}$ is constructed by considering the $I=1 \mathrm{~s}$-channel amplitude and dividing it by $(t-u)$ and then considering the cyclic sum. This amplitude has all the analytic properties of $G_{0}$ and thus all results applicable to it are also applicable to $G_{1} . G_{2}$ is analogously defined after perfoming a further division. [Note that as a result of these divisions, $G_{1}$ and $G_{2}$ in principle verify dispersion relations with fewer subtractions than $G_{0}$.]

The dispersive representation is parameterized by a parameter, $x_{0}$. The crossing symmetric dispersion relations of interest here, arise from first considering dispersion relations in the so-called $x-y$ plane, where the Wanders variables $x$ and $y$, are homogeneous variables of the type $x \sim(s t+t u+u s)$ and $y \sim s t u$. One then writes down dispersion relations on straight lines in the $x-y$ plane given by $y=a\left(x-x_{0}\right)$. A one-parameter family of dispersion relations results, when $a$ is further restricted to be a certain function of $x_{0}$. In Ref. 16] $x_{0}$ is considered in the range, $0 \leq x_{0} \leq 50.41$, in order to generate a closed system of partial wave equations for pion-pion scattering, whose rigorous validity is significantly larger than the original system proposed in Ref.[9]. Note that at fixed $x_{0}$ crossing sym- 
metry does not impose any constraints on the absorptive parts of even the higher waves: constraints are obtained by comparing results at differing $x_{0}$. One may then write down the dispersion relations for this case and extract an expansion in powers of $m_{\pi}^{2}$. We shall first discuss the case of $x_{0}=0$ at some length and shall say a few words about $x_{0} \neq 0$.

\section{1 $x_{0}=0$}

We shall be interested in the treatment of the following dispersion relations for the three amplitudes of interest, for $i=1,2,3$. Furthermore, in the notation of Ref.[16], we would have to supplement the amplitudes $G_{i}$ with overlines: $\bar{G}_{i}$, but for the present work this is redundant and shall be omitted.

$$
\begin{gathered}
G_{i}(s, t, u)=G_{i}(4,0,0)+\frac{1}{\pi} \int_{4 m_{\pi}^{2}}^{\infty} d x \operatorname{Disc} G_{i}(x, \tau(x, a))[x-\tau(x, a)] . \\
\left(2 x+\tau(x, a)-4 m_{\pi}^{2}\right)\left[\frac{1}{\left(x-s m_{\pi}^{2}\right)\left(x-t m_{\pi}^{2}\right)\left(x-u m_{\pi}^{2}\right)}-\frac{1}{x^{2}\left(x-4 m_{\pi}^{2}\right)}\right],
\end{gathered}
$$

where

$$
a=-\frac{s t u}{4(s t+t u+u s)} m_{\pi}^{2}
$$

and

$$
\tau(x, a)=\frac{1}{2}\left[-\left(x-4 m_{\pi}^{2}\right)+\left[\left(x-4 m_{\pi}^{2}\right)^{2}-\frac{16 a}{x+4 a}\left(x\left(x-4 m_{\pi}^{2}\right)\right]^{1 / 2}\right] .\right.
$$

Note the relations for the discontinuities of the three amplitudes in terms of the absorptive parts of the three s-channel iso-spin amplitudes:

$$
\begin{gathered}
\operatorname{Disc}_{0}(s, t)=\frac{1}{3}\left(A^{0}+2 A^{2}\right)(s, t) \\
\operatorname{Disc} G_{1}(s, t)=\frac{1}{6 m_{\pi}^{2}} \frac{3 s-4}{(s-t)(2 s+t-4)}\left(2 A^{0}-5 A^{2}\right)(s, t)+ \\
\frac{1}{m_{\pi}^{2}}\left[\frac{1}{2 t+s-4}-\frac{2 t+s-4}{2(s-t)(2 s+t-4)}\right] A^{1}(s, t)
\end{gathered}
$$




$$
\begin{gathered}
\operatorname{Disc} G_{2}(s, t)=-\frac{1}{2 m_{\pi}^{4}} \frac{1}{(s-t)(2 s+t-4)}\left(2 A^{0}-5 A^{2}\right)(s, t)+ \\
\frac{3}{2 m_{\pi}^{4}} \frac{3 s-4}{(2 t+s-4)(s-t)(2 s+t-4)} A^{1}(s, t)
\end{gathered}
$$

Furthermore, our treatment shall concern only the absorptive parts due to $l \geq 2$ states. As a result, we have the relations:

$$
\begin{gathered}
G_{0}(4,0,0)=0 \\
G_{2}(4,0,0)=\frac{3}{4 m_{\pi}^{2}} G_{1}(4,0,0)=\frac{1}{\pi} \int_{4 m_{\pi}^{2}}^{\infty} d x \frac{2 x-4 m_{\pi}^{2}}{x\left(x-4 m_{\pi}^{2}\right)} \operatorname{Disc}_{2}(x, 0)
\end{gathered}
$$

The s-channel iso-spin amplitudes are now obtained by employing the relations:

$$
\begin{gathered}
T^{0}(s, t, u)=\frac{5}{3} G_{0}(s, t, u)+ \\
\frac{2}{9}\left[(3 s-4) G_{1}(s, t, u) m_{\pi}^{2}-\frac{1}{3}\left(3 s^{2}-16+6 t u\right) G_{2}(s, t, u) m_{\pi}^{4}\right] \\
T^{1}(s, t, u)=\frac{(t-u)}{9}\left(3 G_{1}(s, t, u) m_{\pi}^{2}+(3 s-4) G_{2}(s, t, u) m_{\pi}^{4}\right) \\
T^{2}(s, t, u)=\frac{2}{3} G_{0}(s, t, u)- \\
\frac{1}{9}\left[(3 s-4) G_{1}(s, t, u) m_{\pi}^{2}-\frac{1}{3}\left(3 s^{2}-16+6 t u\right) G_{2}(s, t, u) m_{\pi}^{4}\right]
\end{gathered}
$$

Following the procedure of expanding out the integrands in powers of $m_{\pi}^{2}$ and inserting the narrow-width approximation for the absorptive parts of the partial waves, we first evaluate the contribution of the $f$ to the iso-spin amplitudes resulting from the crossing symmetric (CS) dispersion relations, to this order read:

$$
\begin{gathered}
T^{0}(s, t, u)_{f}^{C S}=\frac{40 \pi \Gamma_{f}^{\pi \pi}}{3 M_{f}^{7}} \sqrt{\frac{M_{f}^{2}}{M_{f}^{2}-4 m_{\pi}^{2}}} m_{\pi}^{4}\left(-32 M_{f}^{2}-192 m_{\pi}^{2}-48 M_{f}^{2} s-496 m_{\pi}^{2} s+\right. \\
\left.14 M_{f}^{2} s^{2}+172 m_{\pi}^{2} s^{2}+2 M_{f}^{2}(t-u)^{2}+12 m_{\pi}^{2}(t-u)^{2}-9 m_{\pi}^{2} s^{3}+17 m_{\pi}^{2} s(t-u)^{2}\right) \\
T^{1}(s, t, u)_{f}^{C S}=\frac{40 \pi \Gamma_{f}^{\pi \pi}}{3 M_{f}^{7}} \sqrt{\frac{M_{f}^{2}}{M_{f}^{2}-4 m_{\pi}^{2}}} m_{\pi}^{4}(t-u)\left(-16 m_{\pi}^{2}-\right. \\
\left.4 M_{f}^{2} s-24 m_{\pi}^{2} s+3 m_{\pi}^{2} s^{2}+m_{\pi}^{2}(t-u)^{2}\right)
\end{gathered}
$$




$$
\begin{gathered}
T^{2}(s, t, u)_{f}^{C S}=\frac{40 \pi \Gamma_{f}^{\pi \pi}}{3 M_{f}^{7}} \sqrt{\frac{M_{f}^{2}}{M_{f}^{2}-4 m_{\pi}^{2}}} m_{\pi}^{4}\left(-32 M_{f}^{2}-192 m_{\pi}^{2}-112 m_{\pi}^{2} s+2 M_{f}^{2} s^{2}+\right. \\
\left.76 m_{\pi}^{2} s^{2}+2 M_{f}^{2}(t-u)^{2}+12 m_{\pi}^{2}(t-u)^{2}-9 m_{\pi}^{2} s^{3}+5 m_{\pi}^{2} s(t-u)^{2}\right)
\end{gathered}
$$

The contribution of the $g$ to the iso-spin amplitudes resulting from the crossing symmetric dispersion relations, to this order read:

$$
\begin{gathered}
T^{0}(s, t, u)_{g}^{C S}=\frac{56 \pi \Gamma_{g}^{\pi \pi}}{M_{f}^{7}} \sqrt{\frac{M_{g}^{2}}{M_{g}^{2}-4 m_{\pi}^{2}}} m_{\pi}^{4}\left(-32 M_{g}^{2}-192 m_{\pi}^{2}+32 M_{g}^{2} s+208 m_{\pi}^{2} s\right. \\
\left.-6 M_{g}^{2} s^{2}-52 m_{\pi}^{2} s^{2}+2 M_{g}^{2}(t-u)^{2}+12 m_{\pi}^{2}(t-u)^{2}+3 m_{\pi}^{2} s^{3}+m_{\pi}^{2} s(t-u)^{2}\right) \\
T^{1}(s, t, u)_{g}^{C S}=\frac{28 \pi \Gamma_{g}^{\pi \pi}}{M_{f}^{7}} \sqrt{\frac{M_{f}^{2}}{M_{f}^{2}-4 m_{\pi}^{2}}} m_{\pi}^{4}(t-u)\left(-16 m_{\pi}^{2}+12 M_{g}^{2} s+72 m_{\pi}^{2} s+\right. \\
\left.m_{\pi}^{2}(t-u)^{2}+3 m_{\pi}^{2} s^{2}\right) \\
T^{2}(s, t, u)_{g}^{C S}=-\frac{1}{2} T^{0}(s, t, u)_{g}^{C S}
\end{gathered}
$$

From the $I=0,2$ iso-spin amplitudes, we now write down

$$
A(s, t, u)_{f}^{C S}=\frac{160 \pi \Gamma_{f}^{\pi \pi}}{3 M_{f}^{7}} \sqrt{\frac{M_{f}^{2}}{M_{f}^{2}-4 m_{\pi}^{2}}} m_{\pi}^{4} s\left(-4 M_{f}^{2}-32 m_{\pi}^{2}+M_{f}^{2} s+8 m_{\pi}^{2} s+m_{\pi}^{2}(t-u)^{2}\right)
$$

and

$$
\begin{gathered}
A(s, t, u)_{g}^{C S}=\frac{28 \pi \Gamma_{g}^{\pi \pi}}{M_{g}^{7}} \sqrt{\frac{M_{g}^{2}}{M_{g}^{2}-4 m_{\pi}^{2}}} m_{\pi}^{4}\left(-32 M_{g}^{2}-192 m_{\pi}^{2}+32 M_{g}^{2} s+208 m_{\pi}^{2} s-\right. \\
\left.6 M_{g}^{2} s^{2}-52 m_{\pi}^{2} s^{2}+2 M_{g}^{2}(t-u)^{2}+12 m_{\pi}^{2}(t-u)^{2}+3 m_{\pi}^{2} s^{3}+m_{\pi}^{2} s(t-u)^{2}\right)
\end{gathered}
$$

The expressions in eq.(4.15) and eq.4.16) constitute some of the most important results in this work. Also note the striking feature that the amplitude $A(s, t, u)_{g}^{C S}$ is identical in structure to eq. (3.5) $A(s, t, u)_{\rho}$, upto their respective angular momentum multiplicites of 7 and 3 respectively.

A check to our results arises from considering the sum of the contributions from the resonances $f$ and $g^{\prime}$, where $g^{\prime}$ is a hypothetical resonance whose mass is equal to that of 
the $g$, but whose width is tuned to agree with eq.(3.12). The sum of these amplitudes from fixed-t dispersion relations, viz., eq.(3.6)-eq.(3.8) and eq.(3.9)-eq.(3.11) the latter evaluated with the width given by eq. (3.12) is identical to those from the crossing symmetric dispersion relations, viz., eq.(4.9)-eq.(4.11) and eq.(4.12)-eq.(4.14) the latter evaluated with the width given by eq.(3.12). This is due to crossing constraints being satisfied by the former in this event.

\section{$4.2 \quad x_{0} \neq 0$}

The dispersion relations for $x_{0} \neq 0$ for the $G_{i}$ turn out to be far more complicated. Furthermore, the analog of eq.(4.2) for non-zero $x_{0}$ is now:

$$
a\left(x_{0}\right)=-\frac{s t u}{4\left(s t+t u+u s+16 x_{0}\right)} m_{\pi}^{2} .
$$

Furthermore, the are expressions analogous to $\tau(a)$ of the previous subsection and dispersive representation for the $G_{i}$ has been listed explicitly in Ref.[16]. We have considered these representations and worked out the contributions of our resonances at $O\left(m_{\pi}^{6}\right)$. Consider for instance, the contribution of the $f$ at $O\left(m_{\pi}^{6}\right)$ to the low-energy expansion:

$$
\begin{gathered}
A(s, t, u)_{f}^{C S, x_{0}}=\frac{160 \pi \Gamma_{f} \sqrt{\frac{M_{f}^{2}}{M_{f}^{2}-4 m_{\pi}^{2}}} m_{\pi}^{4}(-4+s) s}{3 M_{f}^{5}}+ \\
\frac{160 \pi \Gamma_{f} \sqrt{\frac{M_{f}^{2}}{M_{f}^{2}-4 m_{\pi}^{2}}} m_{\pi}^{6} s}{3 M_{f}^{7}\left(16+8 s-3 s^{2}-(t-u)^{2}+16 x_{0}\right)} \cdot\left(-512-128 s+160 s^{2}-24 s^{3}+48(t-u)^{2}-\right. \\
\left.3 s^{2}(t-u)^{2}-(t-u)^{4}-1216 x_{0}+480 s x_{0}-44 s^{2} x_{0}+60(t-u)^{2} x_{0}\right)
\end{gathered}
$$

While with $x_{0}=0$, the low-energy polynomial was produced by the repeated use of the identity $s+t+u=4$, for the case at hand, the appearance of the parameter $x_{0}$ prevents the factorizing of a series in $m_{\pi}^{2}$ into a sum of terms that is a polynomial in $s$ and $(t-u)^{2}$ 
for $A(s, t, u)$. The result above illustrates our remark. We also note that the answer at $O\left(m_{\pi}^{4}\right)$ is independent of $x_{0}$ as expected. Furthermore, it may be easily checked that in the limit of $x_{0} \rightarrow 0$, we recover our results of the previous subsection. It appears, therefore, that only the choice with $x_{0}=0$ can produce a low-energy polynomial that is manifestly crossing symmetric.

The analogous result for the $g$ at this order in $m_{\pi}$ corresponds to the $x_{0}=0$ answer even at non-zero $x_{0}$. The dependence on this parameter manifests itself at the next order.

\section{$5 \quad$ Numerical Results}

We discuss the numerical import of our work in this section. We first discuss the case of resonance saturation at $O\left(p^{4}\right)$. It may be seen by inspection that at this order no crossing constraints arise. As a result, we may unambiguously compute the contributions of the $\rho, f$ and $g$ respectively. The primary reason for our consideration of the $\rho$ is that it sets the scale for the contributions that arise from the S- and P-wave absorptive parts. Crossing symmetry dictates that the presence of the $\rho$ necessarily implies contributions to the low-energy polynomials of a similar order of magnitude from the S- waves; here we do not embark on a detailed numerical analysis. We insert the values of the parameters listed in Table 1, with $\Gamma^{\pi \pi}$ given by $\Gamma_{t o t} \cdot B F$, where $\Gamma_{t o t}$ is the total width and $B F$ is the $\pi \pi$ branching fraction, into the terms of $O\left(p^{4}\right)$ of eq.(3.5), eq.(3.16) and eq.(3.17). Comparing with eq.(2.10), we isolate the $\beta_{i}, i=1,2,3,4$ and tabulate the results in Table 2. An inspection of the Table 2 shows that at $O\left(p^{4}\right)$ the contributions from the $f$ certainly cannot be neglected even in comparison with the contribution of the $\rho$. The contribution of 
the $g$ is an order of magnitude lower to $\beta_{i}$. It is then a standard exercise to translate these results and read off the contributions of the resonances to the the one-loop chiral coupling constants, the $\overline{l_{i}}, i=1,2$, which are also tabulated in Table 2. Pion-pion scattering also involves the coupling constants $\overline{l_{i}}, i=3,4$. Resonance saturation does not contribute to these coupling constants at this order. It is also instructive to compare our results with those from effective lagrangian comptuations of Ref.[20, 21, 10] which are found to be in agreement, when the constraints from the Froissart bound are enforced on the latter.

At $O\left(p^{6}\right)$ we compile the contributions of the resonances of interest to the $\beta_{i}$. This comparison is performed between eq.(3.5), eq.(3.16), eq.(3.17) and eq.(2.10) and then between eq.(3.5), eq.(4.15), eq.(4.16) and eq.(2.10), upon inserting the values of the parameters listed in Table 1. This procedure yields the results tabulated in Tables 3 and 4 respectively. One sees that the contributions from the fixed-t and the crossing symmetric dispersion relations have important and interesting discrepancies. However, if the width of the $g$ is tuned to satisfy eq. (3.12), (we have referred to such a hypothetical resonance as the $g^{\prime}$ ), then the sum of the contributions of $f$ and $g^{\prime}$ to each of the $\beta_{i}$ is the same no matter if we compute them from the fixed-t or the crossing symmetric dispersion relations as indeed it should be.

It turns out that the requirment that crossing symmetry be restored at this order by the $f$ and the $g$, eq.(3.12), with the known masses of the $f$ and $g$ and the width of the $f$ from Table 1, predicts for the $\pi \pi$ width of the $g \Gamma_{g}^{\pi \pi}$, the value of $109 \mathrm{MeV}$. This is to be compared with its experimental value of $38 \mathrm{MeV}$. It is precisely this problem which led us to consider the crossing symmetric representations: the problem of the mismatch between the "predicted" and observed widths of the $g$ resonance is evaded by considering resonance 
saturation with manifestly crossing symmetric dispersion relations. From Table 4 we can unambiguously read off the contributions of the $f$ and the $g$ to the low-energy polynomials. Thus the results of Table 4 constitute the central results in this work. Somewhat improved values for the $\beta_{i}$ can be obtained by replacing the narrow-width approximation by other phenomenological models of these and higher partial waves. Since the resonances considered here essentially dominate the pion-pion phenomenology, the present work gives a realistic estimate of the contributions of the relevant partial waves.

\section{Conclusions}

In this work, we have shown that $l \geq$ states contribute to the low-energy polynomial in a manner that can be computed explicitly from the dispersion relations with two subtractions. Whereas fixed-t dispersion relations require us to satisfy crossing constraints, the problem can be avoided through the use of manifestly crossing symmetric dispersion relations. We have shown that, since the width of the $g$ is smaller by a factor of nearly 3 than the requirement of crossing constraints of the fixed-t dispersion relations, it is practical to evaluate the contributions of the $f$ and $g$ states using the crossing symmetric dispersion relations. We conclude with the remark that the subtle property of crossing symmetry in field theory and dispersion relations must be treated with great care in matters of principle and practice.

Acknowledgements: It is a pleasure to thank Prof. H. Leutwyler for discussions. I also thank P. Büttiker and D. Toublan for a careful reading of the manuscript, and D. Toublan for useful correspondence on tensor meson dominance. 


\section{References}

[1] J. Gasser and H. Leutwyler, Ann. Phys. 158, 142 (1984).

[2] J. Stern, H. Sazdjian, and N. H. Fuchs, Phys. Rev. D 47, 3814 (1993).

[3] M. Knecht, B. Moussallam, J. Stern, and N. H. Fuchs, Nucl. Phys. B 457, 513 (1995).

[4] J. Bijnens, G. Colangelo, G. Ecker, J. Gasser, and M. E. Sainio, Phys. Lett. B 374, 210 (1996).

[5] J. Bijnens, G. Colangelo, G. Ecker, J. Gasser, and M. E. Sainio, Nucl. Phys, 508, $263(1997)$.

[6] M. M. Nagels et al., Nucl. Phy. B147, 189 (1979).

[7] For a review, see H. Leutwyler, "Probing the quark condensate by means of $\pi \pi$ scattering, preprint, hep-ph/9709406, (1997).

[8] G. Wanders, Springer Tracts in Modern Physics 57, pp 22-40, G. Höhler, ed., Springer-Verlag, Berlin (1971).

[9] S. M. Roy, Phys. Lett. 36 B, 353 (1971).

[10] H. Leutwyler, et al., to be published.

[11] J.-L. Basdevant, J. C. Le Guillou, and H. Navelet, Nuovo Cimento A 7, 363 (1972).

[12] B. Ananthanarayan and P. Büttiker, Phys. Rev. D 54, 1125 (1996). 
[13] M. Knecht, B. Moussallam, J. Stern, and N. H. Fuchs, Nucl. Phys. B 471, 445 (1996).

[14] Particle Data Group, R. M. Barnett et al., Phys. Rev. D 54, 1 (1996).

[15] G. Wanders, Phys. Lett. 19, 331 (1965); Helv. Phys. Acta, 39, 228 (1966).

[16] G. Mahoux, S. M. Roy, and G. Wanders, Nucl. Phys. B 70, 297 (1974).

[17] G. Wanders, Phys. Rev. D 56, 4328 (1997).

[18] G. Ecker, J. Gasser, H. Leutwyler, A. Pich and E. de Rafael, Phys. Lett. B223, 425 (1989).

[19] B. Ananthanarayan and P. Büttiker, Phys. Lett. B415, 402 (1997).

[20] D. Toublan, Phys. Rev. D 53, 6602 (1996); erratum (to be published).

[21] S. Bellucci, J. Gasser, and M.. E. Sainio, Nucl. Phys. B423, 80 (1994). 


\section{Table Captions}

1. Iso-spin, angular momenta, masses, widths (in $\mathrm{MeV}$ ) and $\pi \pi$ branching fractions used in our numerical results for the $\rho, f$ and $g$ resonances.

2. Contributions of the $\rho, f$ and $g$ resonances to $\beta_{1,2,3,4}$ at $O\left(p^{4}\right)$ and the corresponding contributions to $\bar{l}_{1,2}$. We have taken $m_{\pi}=139.6 \mathrm{MeV}$ and $F_{\pi}=92.4 \mathrm{MeV}$.

3. Contributions of the $\rho, f$ and $g$ resonances to $\beta_{1,2,3,4,5,6}$ at $O\left(p^{6}\right)$ from fixed-t dispersion relations.

4. Contributions of the $\rho, f$ and $g$ resonances to $\beta_{1,2,3,4,5,6}$ at $O\left(p^{6}\right)$ from crossing symmetric dispersion relations. 


\begin{tabular}{|c|c|c|c|c|c|}
\hline$I$ & $l$ & $m$ & $M$ & $\Gamma_{\text {tot }}$ & $B F$ \\
\hline 1 & 1 & $\rho$ & 769 & 151 & $100 \%$ \\
\hline 0 & 2 & $f$ & 1275 & 185 & $85 \%$ \\
\hline 1 & 3 & $g$ & 1690 & 160 & $24 \%$ \\
\hline
\end{tabular}

Table 1

\begin{tabular}{|c|c|c|c|c|c|c|}
\hline$m$ & $\beta_{1}$ & $\beta_{2}$ & $\beta_{3}$ & $\beta_{4}$ & $\bar{l}_{1}$ & $\bar{l}_{2}$ \\
\hline$\rho$ & -0.276 & 0.276 & -0.0517 & 0.0172 & -6.27 & 3.13 \\
\hline$f$ & 0 & -0.0122 & 0.00305 & 0 & 0.277 & 0 \\
\hline$g$ & -0.0030 & 0.0030 & $-5.66 \cdot 10^{-4}$ & $1.88 \cdot 10^{-4}$ & -0.069 & 0.034 \\
\hline
\end{tabular}

Table 2 


\begin{tabular}{|c|c|c|c|c|c|c|}
\hline$m$ & $\beta_{1}$ & $\beta_{2}$ & $\beta_{3}$ & $\beta_{4}$ & $\beta_{5}$ & $\beta_{6}$ \\
\hline$\rho$ & -0.330 & 0.335 & -0.0665 & 0.0210 & $8.54 \cdot 10^{-4}$ & $2.84 \cdot 10^{-4}$ \\
\hline$f$ & 0 & -0.0136 & 0.00348 & 0 & $-1.82 \cdot 10^{-5}$ & $5.47 \cdot 10^{-5}$ \\
\hline$g$ & -0.00314 & 0.00326 & $-6.51 \cdot 10^{-4}$ & $1.96 \cdot 10^{-4}$ & $8.36 \cdot 10^{-6}$ & $-5.79 \cdot 10^{-6}$ \\
\hline
\end{tabular}

Table 3

\begin{tabular}{|c|c|c|c|c|c|c|}
\hline$m$ & $\beta_{1}$ & $\beta_{2}$ & $\beta_{3}$ & $\beta_{4}$ & $\beta_{5}$ & $\beta_{6}$ \\
\hline$\rho$ & -0.330 & 0.335 & -0.0665 & 0.0210 & $8.54 \cdot 10^{-4}$ & $2.84 \cdot 10^{-4}$ \\
\hline$f$ & 0 & -0.0133 & 0.00333 & 0 & 0 & $3.65 \cdot 10^{-5}$ \\
\hline$g$ & -0.00314 & 0.00315 & $-5.99 \cdot 10^{-4}$ & $1.96 \cdot 10^{-4}$ & $1.93 \cdot 10^{-6}$ & $6.43 \cdot 10^{-7}$ \\
\hline
\end{tabular}

Table 4 